\title{
PER-NODE POWER MINIMAL MULTICAST TREES WHICH MAXIMIZE THE TIME-TO-FIRST-FAILURE IN ENERGY CONSTRAINED STATIC WIRELESS NETWORKS : A 2-STEP HEURISTIC APPROACH
}

\author{
Arindam K. Das \\ Department of Electrical Engineering \\ University of Washington, Box 352500, Seattle, WA 98195. \\ email: arindam@ee.washington.edu \\ Payman Arabshahi \\ Jet Propulsion Laboratory \\ 4800 Oak Grove Drive, MS 238-343, Pasadena, CA 91109. \\ email: payman@jpl.nasa.gov
}

\begin{abstract}
We consider the problem of jointly maximizing the time-tofirst-failure (TTFF), defined as the time till the first node in the network runs out of battery energy, and minimizing the total power in energy constrained static wireless networks. It is shown in [6] that simply optimizing the TTFF criterion may not provide the ideally optimum solution. Besides maximizing the TTFF, the ideally optimum solution guarantees that the lifetimes of all nodes are at least as high as in other trees which provide the same TTFF. A composite objective function involving the TTFF and the sum of the transmitter powers is therefore proposed in [6], along with a discussion of an optimal solution methodology using mixed integer linear programming. In this paper, we discuss a 2-step heuristic procedure for the joint optimization problem. The first step is a greedy iterative algorithm which provides an optimal solution, but with respect to the TTFF criterion only. The second step is a tree-improvement technique which is used to refine the TTFF-optimal tree such that the total transmitter power is minimized, without affecting the optimal TTFF. Recent work has shown that the power consumed in the receiver circuitry can be almost comparable to the transmit power, especially in short-range networks. Our algorithms are therefore designed to take into account both the transmitter side and receiver side power expenditures. Simulation results are presented to validate the performance of the algorithm.
\end{abstract}

\section{INTRODUCTION}

We consider the problem of jointly maximizing the timeto-first-failure (TTFF), defined as the time till the first node in the network runs out of battery energy, and minimizing the total transmit power in energy constrained static wireless networks. In applications where replacement/maintenance of such batteries is difficult or infeasible, it is of utmost importance to design routing protocols which maximize the lifetime of the network. A metric commonly used to define the lifetime of a network is the duration of time before any node in the network runs out of its battery energy. We define this time to be the time-to-first-failure (TTFF), also known as system lifetime or network lifetime in the literature. To the best of our knowledge, this problem was first addressed by Chang and Tassiulas for an unicast application [1]. Subsequent research in this area for unicast as well as multicast applications has been reported in [2], [3], [4] and [5].

It is shown in [6] that simply optimizing the TTFF criterion may not provide the ideally optimum solution. Besides maximizing the TTFF, the ideally optimum solution guarantees that the lifetimes of all nodes are at least as high as in other trees which provide the same TTFF. A composite objective function involving the TTFF and the sum of the transmitter powers is therefore suggested in [6]. In this paper, we discuss a 2-step heuristic procedure for solving the joint optimization problem. For a multicast application, the first step is the $M D L T$ algorithm [7], which is followed by a treeimprovement phase. This latter phase aims to reduce the total transmitter power of the tree, without worsening the TTFF.

The rest of the paper is organized as follows. We outline the network model in Section II, which is followed by a brief definition of the problem in Section III. A brief review of the $M D L T$ algorithm is provided in Section IV. In Section V, we discuss the improvement procedure for refining the $M D L T$ tree. Simulation results are presented in Section VI. 


\section{NeTwORK MOdeL}

We assume a fixed $N$-node network with a specified source node which has to broadcast (multicast) a message to all (some) other nodes in the network. We assume static broadcasting (multicasting); i.e., the same tree is used for the entire broadcast (multicast) duration. Any node can be used as a relay node to reach other nodes in the network. All nodes are assumed to have omni-directional antennas, so that if node $i$ transmits to node $j$, all nodes closer to $i$ than $j$ will also receive the transmission (provided line-of-sight exists).

We assume that, for a transmission from node $i$ to $j$, the received signal power at $j$ varies as $d_{i j}^{-\alpha}$, where

$$
d_{i j}=\left[\left(x_{i}-x_{j}\right)^{2}+\left(y_{i}-y_{j}\right)^{2}\right]^{1 / 2}
$$

is the Euclidean distance between nodes $i$ and $j,\left(x_{i}, y_{i}\right)$ are the coordinates of node $i$ and $\alpha$ (typically in the range $2 \leq \alpha \leq 4)$ is the channel loss exponent. Consequently, the transmitter power at $i$ necessary to support the link $(i \rightarrow j)$, $\mathbf{P}_{i j}$, is proportional (accounting for link and antenna gains) to $d_{i j}^{\alpha}$. Without any loss of generality, we set the proportionality constant to be equal to 1 and therefore:

$$
\mathbf{P}_{i j}=d_{i j}^{\alpha}
$$

The transmit power matrix of a network, $\mathbf{P}$, is an $N \times N$ symmetric matrix whose $(i, j)$ th element, $\mathbf{P}_{i j}$, represents the transmission power required at node $i$ to support the link $(i \rightarrow j)^{1}$. We denote the set of all nodes in the network by $\mathcal{N}$ and $N=|\mathcal{N}|$.

In order to capture the effect of the power expenditure at the receiver circuitry on the node lifetimes, we introduce a link cost matrix, $\mathbf{C}_{i j}$, whose $(i, j)$ th element, $\mathbf{C}_{i j}$, is interpreted as the total cost of activating the link $(i \rightarrow j)$. Specifically, $\mathbf{C}_{i j}$ comprises a transmitter power cost at $i, \mathbf{P}_{i j}(1)$, and a receiver power cost at $j$, denoted by $P_{j}^{r x}$ :

$$
\mathbf{C}_{i j}=d_{i j}^{\alpha}+P_{j}^{r x}=\mathbf{P}_{i j}+P_{j}^{r x}
$$

Strictly speaking, there is also an additional power consumption in the transmitter circuitry. We assume that this term is negligible compared to the antenna power, $d_{i j}^{\alpha}$.

It should be noted that if the elements of the transmit power matrix, $\mathbf{P}$, satisfy the triangle inequality (which it may not because of the exponent $\alpha$ ) - i.e., for any 3 nodes $i, j$ and $k, \mathbf{P}_{i j}+\mathbf{P}_{j k} \geq \mathbf{P}_{i k}$ - and if $P_{j}^{r x}$ is identical for all nodes $j \in \mathcal{N}$, the optimal solution will be the same whether or

\footnotetext{
${ }^{1}$ In this paper, we assume that all links (edges) are directed. The notation $(i \rightarrow j)$ will be used to denote a directed edge from node $i$ to $j$. The notation $(i, j)$ will be used to refer to the node pair.
}

not the receiver power component is included in (2). If the triangle inequality is not satisfied, the optimal solution may vary significantly depending on whether the receiver power component is factored in or not, as illustrated in Figure 1.

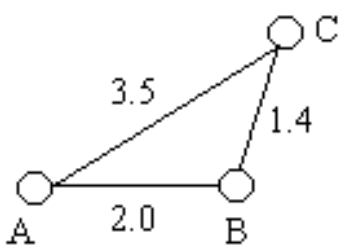

(a)

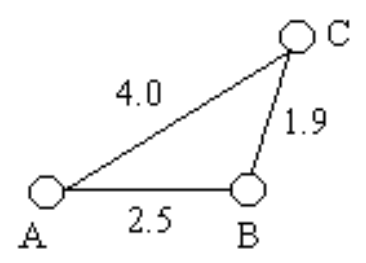

(b)
Fig. 1. (a) The costs of the links represent the $\mathbf{P}_{i j} \mathbf{s}$ and do not satisfy the triangle inequality. The least cost path from node $A$ to $C$ is the 2-hop path $A \rightarrow B \rightarrow C$. (b) The costs of the links, including a receiver power component, $P_{j}^{r x}=0.5$, for all nodes $j$. In this case, the least cost path from $A$ to $C$ is the 1-hop path $A \rightarrow C$.

\section{Problem Statement}

Let $E(t)$ be a vector of node residual energies at time $t$, the ith element of $E(t)$ representing the residual energy of node $i$ at time $t$, and $Y$ be a vector of average node powers. The element $Y_{i}$ represents the average power expenditure of node $i$ and is defined as follows:

$$
Y_{i}=Y_{i}^{t x}+Y_{i}^{r x}
$$

where $Y_{i}^{t x}$ and $Y_{i}^{r x}$ represent the power consumption at nodes $i$ and $j$ due to transmission and reception activities receptively. Define $\mathbf{X}_{i j}$ to be a binary variable which is equal to 1 if the link $(i \rightarrow j$ ) is chosen in the optimal solution and 0 otherwise. Then, the components $Y_{i}^{t x}$ and $Y_{i}^{r x}$ are given by:

$$
\begin{aligned}
Y_{i}^{t x} & =\max \left(\mathbf{X}_{i j} \mathbf{P}_{i j}: j \in \mathcal{N}, j \neq i\right) \\
Y_{i}^{r x} & =\max \left(\mathbf{X}_{j i} \mathbf{P}_{i}^{r x}: j \in \mathcal{N}, j \neq i\right)
\end{aligned}
$$

We assume that each node has a constraint on maximum transmitter power, denoted by $P_{i}^{\max }$. That is, $Y_{i}^{t x} \leq P_{i}^{\max }$, $\forall i \in \mathcal{N}$.

Also, let $s$ denote the source, $\mathcal{E}$ the set of all directed edges and $\mathcal{D}$ the set of destination nodes, $\mathcal{D} \subseteq \mathcal{N} \backslash s$. The cardinalities of $\mathcal{E}$ and $\mathcal{D}$ are $E$ and $D$ respectively; i.e., $E=|\mathcal{E}|$ and $D=|\mathcal{D}|$. Using the transmitter power constraint, the set of all edges, $\mathcal{E}$, is given by:

$$
\mathcal{E}=\left\{(i \rightarrow j):(i \neq j) \in \mathcal{N}, Y_{i}^{t x} \leq P_{i}^{\max }, j \neq s\right\}
$$

Defining $L_{i}(t) \triangleq E_{i}(t) / Y_{i}$ to be the lifetime of node $i$, the problem of maximizing the TTFF can be written as:

$$
\text { maximize }\left\{\min _{i \in \mathcal{N}} L_{i}(t)\right\}
$$


Note that the value of the expression within curly braces in (7) is dependent on the time index $t$ and hence, strictly speaking, should be termed residual-time-to-first-failure. However, we will refer to it simply as the time-to-firstfailure, implicitly recognizing its dependence on the time origin $t$. Accordingly, henceforth in this paper, we will simply use the notations $E_{i}$ and $L_{i}$ instead of $E_{i}(t)$ and $L_{i}(t)$. We now express the objective function in (7) as a minimax optimization problem as shown below:

$$
\begin{aligned}
\operatorname{maximize}\left(\min _{i} L_{i}\right) & =\operatorname{maximize}\left(\min _{i} E_{i} / Y_{i}\right) \\
& =\operatorname{minimize}\left(\max _{i} Y_{i} / E_{i}\right) \\
& =\operatorname{minimize} \sigma
\end{aligned}
$$

where $\sigma=\max _{i}\left(Y_{i} / E_{i}\right)=1 / \tau$ and $\tau$ is the TTFF.

As explained in [6], simply optimizing the TTFF criterion may not yield the best possible solution. Specifically, it was shown that the transmitter power levels of all nodes are not guaranteed to be minimal, thereby leading to a reduced average node lifetime. We therefore proposed the use of a joint objective function involving the TTFF criterion and the sum of average node powers ${ }^{2}$ :

$$
\text { minimize } w_{1} \sigma+w_{2} \sum_{i=1}^{N} Y_{i}
$$

where $\sum_{i=1}^{N} Y_{i}$ is the sum of average node powers and $\left\{w_{1}, w_{2}\right\}$ are suitably chosen non-negative penalty factors. The first parameter in (10), $\sigma$, is viewed as the global cost while the second parameter, $\sum_{i=1}^{N} Y_{i}$, is viewed as the sum of local costs.

In this paper, we are concerned primarily with the case when $w_{1}>>w_{2} \neq 0$. For a proper choice of these parameter values, it is possible to obtain the "best possible" tree which maximizes the TTFF while ensuring that the solution is the most power efficient among the set of all trees with optimal TTFF. In the following sections, we describe a 2-step heuristic procedure for solving this special case of the general optimization problem (10). The first step is the MDLT algorithm [7], which is followed by a tree-improvement phase which aims to reduce the total transmitter power of the tree, without worsening its TTFF.

\section{BRief Review OF THE MDLT Algorithm}

\section{A. Notation}

$$
k \quad=\text { iteration number }
$$

\footnotetext{
${ }^{2}$ The optimization model in [6] considered transmit powers only. However, it can be easily modified to account for power consumption due to reception if $Y_{i} \mathrm{~s}$ are defined as in (3).
}

$$
\begin{array}{ll}
T^{k} & =\text { connection tree grown till iteration } k \\
\tau^{k} & =\text { lifetime of the partially grown tree } T^{k} \\
\mathbf{N R}^{k} & =\text { all nodes reached till iteration } k \\
\mathbf{N N R}^{k} & =\text { nodes not reached at the end of iteration } k \\
& \triangleq \mathcal{N} \backslash \mathbf{N} \mathbf{R}^{k}
\end{array}
$$

\section{B. The algorithm}

In this section, we briefly review the minimum decremental lifetime (MDLT) algorithm for optimizing the objective function in (10) with $w_{2}=0$. A detailed description can be found in [7]. While the algorithm in [7] considers transmit powers only, the review here generalizes the procedure to include both transmit and receive powers. The proof of optimality provided in [7] extends straightforwardly when both transmit and receive powers are accounted for.

Procedure MDLT is an iterative greedy algorithm wherein one new node is spanned into the tree at each iteration. For $k=0$, we initialize:

$$
\mathbf{N R}^{0}=s, \mathbf{N N R}^{0}=\mathcal{N} \backslash \mathbf{N R}^{0}, T^{0}=\emptyset, \tau^{0}=0
$$

For any $k \geq 1$, an edge is chosen from a list of candidate edges defined as follows:

$$
\begin{array}{r}
\text { edge_list }^{k}=\left\{(i \rightarrow j): \forall i \in \mathbf{N R}^{k-1}, \forall j \in \mathbf{N N R}^{k-1},\right. \\
\left.\left(E_{i} / \mathbf{P}_{i j}, E_{j} / \mathbf{P}_{j}^{r x}\right) \geq L / R,(i \rightarrow j) \in \mathcal{E}\right\}
\end{array}
$$

where $L$ is the total number of bits to be transmitted during the multicast session and $D$ is the data rate in bits per second. The first two conditions in (12) allow transmissions from any node which has been spanned into the tree by iteration $k-1$, to any node not yet spanned in. The third condition in (12), which is to be interpreted as requiring both $E_{i} / \mathbf{P}_{i j} \geq L / R$ and $E_{j} / \mathbf{P}_{j}^{r x} \geq L / R$, is the session duration support criterion and prevents nodes lacking sufficient battery capacity from participating in the multicast tree as a transmitter or a receiver, or both.

The edge $(i \rightarrow j$ ) is chosen to be included in the connection tree at iteration $k$ if:

$$
\min \left(\frac{E_{i}}{\mathbf{P}_{i j}}, \frac{E_{j}}{\mathbf{P}_{j}^{r x}}\right)<\min \left(\frac{E_{m}}{\mathbf{P}_{m n}}, \frac{E_{n}}{\mathbf{P}_{n}^{r x}}\right)
$$

where $(i \rightarrow j) \in$ edge_list ${ }^{k},(m \rightarrow n) \in$ edgellist $^{k}$ and $(m \rightarrow n) \neq(i \rightarrow j)$. After the edge is chosen, the sets $\mathbf{N R}^{k}$ and $\mathbf{N N R}^{k}$ are updated as follows:

$$
\mathbf{N R}^{k}=\mathbf{N R}^{k-1} \cup j, \mathbf{N N R}^{k}=\mathcal{N} \backslash \mathbf{N} \mathbf{R}^{k}
$$


The lifetime of the connection tree after inclusion of the edge $(i \rightarrow j)$ at iteration $k$ is given by:

$$
\tau^{k}=\min \left(\tau^{k-1}, E_{i} / \mathbf{P}_{i j}, E_{j} / \mathbf{P}_{j}^{r x}\right)
$$

The algorithm terminates when all destination nodes are reached. The worst case time complexity (corresponding to a broadcast application) of the algorithm is $O\left(|N|^{2}\right)$.

\section{Pruning the MDLT tree}

Given a directed graph, $G$, the descendants of node $i$, denoted by $d e(i)$, is defined as the set of nodes, $\{j\}$, such that there is a path from $i$ to all nodes in $\{j\}$. That is,

$$
\operatorname{de}(i) \triangleq\{j \mid i \mapsto j \text { but not } j \mapsto i\}
$$

where $(i \mapsto j)$ is a directed path from node $i$ to node $j$. Conversely, the non-descendants of node $i$, denoted by $n d(i)$, is defined as:

$$
n d(i) \triangleq \mathcal{N} \backslash\{i \cup \operatorname{de}(i)\}
$$

For a multicast application, the MDLT tree can have a lot of redundant edges. An edge, $(i \rightarrow j)$, is deemed to be redundant if $j$ itself is not a destination node or none of the descendants of $j$ is a destination node. The MDLT tree must therefore be pruned to eliminate all redundant edges before the tree-improvement algorithm is applied. Note that the pruning step itself may lead to a reduction of total transmitter power in the tree, which can be quite substantial if $D / N<<1$.

\section{Algorithm FOR improving MDLT treES}

As mentioned in the previous section, the MDLT algorithm generates optimal trees, but only with respect to the TTFF criterion. In this section, we explain a variant of the 1-shrink algorithm ${ }^{3}$ which can be used to reduce the total transmitter power of the MDLT tree "without worsening the TTFF". We name this algorithm 1-shrink for MDLT.

Given a transmission from node $i$ to node $j$, with nodes $\alpha_{0}, \alpha_{1}, \cdots$ and $\alpha_{k}$ covered implicitly, let $\left\{\alpha_{0}, \alpha_{1}, \cdots, \alpha_{k}\right.$, $j\}$ be an ordering of the nodes with respect to their distance from $i$. That is, $\alpha_{0}$ is closest to $i, \alpha_{1}$ is second closest, $\cdots$ and $j$ is the farthest from $i$. The 1 -shrink operation applied to node $i$ implies a reduction of its transmission power level (or, shrinkage of its transmission radius) by 1 notch, such that the farthest node now reached is $\alpha_{k}$ instead of $j$. For example, applying the 1-shrink operation to node 4 in Figure 2(a) would result in it transmitting to node 3 , leaving node 1 disconnected.

\footnotetext{
${ }^{3}$ The original algorithm was proposed in [8] for refining sub-optimal minimum power broadcast trees.
}

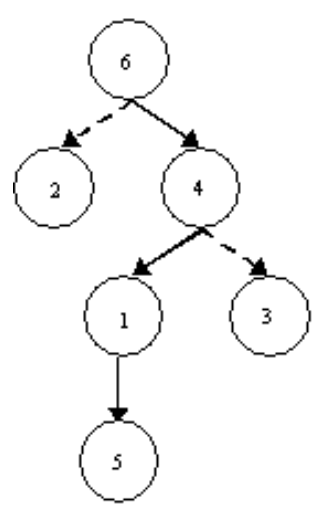

(a)

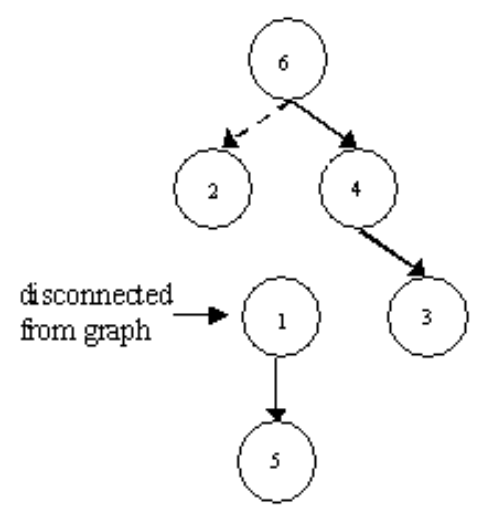

(b)
Fig. 2. (a) Example multicast tree. Node 6 is the source. The solid lines imply actual transmissions and the dotted lines imply implicit transmissions. For example, $6 \rightarrow 2$ is an implicit transmission since node 2 is closer to node 6 than 4 . In this tree, node 6 (the source) is said to be at level 0 , nodes 2 and 4 at level 1 , nodes 1 and 3 at level 2 , and node 5 at level 3. Clearly, if the maximum level in a digraph is $M A X_{-} L V L$, all nodes at level $l=M A X \_L V L$ will be leaf nodes. Conversely, the maximum level parent nodes in such a digraph can occupy is $M A X_{-} L V L-1$. (b) Result of applying the 1 -shrink operation to node 4 . Note that $(4 \rightarrow 3)$ is now an actual transmission.

Clearly, the 1-shrink operation can be applied only on parent nodes in a digraph. Since our intention is to improve the MDLT tree without worsening the TTFF, we exclude the critical node of the MDLT tree from the set of parent nodes to which the 1-shrink operation can be applied. Given the optimality of the MDLT algorithm, it should not be possible to improve the TTFF by shrinking the transmission radius of the critical node and assigning a new parent (discussed subsequently) to the temporarily disconnected child. Additionally, the 1-shrink operation is applied to the source node only if its number of children is greater than 1 . This prevents the possibility of the source being a leaf in the graph. The set of candidate parent nodes in a digraph which can be subjected to the 1-shrink operation is therefore:

- $\{$ all parent nodes $\} \backslash$ cr_node ${ }_{M D L T}$, if $|\operatorname{ch}(s)|>1$.

- $\{$ all parent nodes $\} \backslash\left\{c r_{\_}\right.$node $\left.e_{M D L T} \cup s\right\}$, otherwise.

where $c r_{-} n o d e_{M D L T}$ is the critical node of the MDLT tree and $\operatorname{ch}(s)$ is the set of all children of the source. For example, in Figure 2(a), $\operatorname{ch}(s)=\operatorname{ch}(6)=\{2,4\}$.

Given the pruned MDLT tree, represented as a digraph rooted at the source, the algorithm works by sequentially applying the 1-shrink operation to the parent nodes in the graph and checking whether the children, which have been temporarily disconnected from the graph as a result of the shrinkage operation, can be better accommodated from any of their non-descendants, excluding the current parent and the critical node of the MDLT tree. If node $i$ is a child of 
$j$, the set of the non-descendants of $i$, excluding its current parent and the critical node of the MDLT tree, is given by $n d(i) \backslash\left\{j \cup c r \_\right.$node $\left.M D L T\right\}$. We will refer to members of such a set as the foster parents of node $i$, denoted by $f p a(i){ }^{4}$ Therefore:

$$
f p a(i) \triangleq n d(i) \backslash\left\{p a(i) \cup c r \_n o d e_{M D L T}\right\}
$$

For example, assuming that node 6 is the critical node in Figure 2, $f p a(1)=n d(1) \backslash\left\{p a(1) \cup c r \_n o d e_{M D L T}\right\}=$ $\{2,3,4,6\} \backslash\{4,6\}=\{2,3\}$.

The criteria used to determine whether a temporarily disconnected child retains its existing parent or is assigned a new parent from the set of its foster parents are: (1) incremental and decremental transmit power costs (2) TTFF violation (3) maximum transmitter power constraint and (4) session duration support. Criteria (2), (3) and (4) are required since the tree-improvement algorithm may increase the transmitter power of certain nodes. We describe each of the criteria below.

- For a node $i$ transmitting to node $j$, the incremental transmit power cost of adding node $k$ to its reach is $\mathbf{P}_{i k}-\mathbf{P}_{i j}$. If $i$ is a non-transmitting node, the incremental cost of adding node $k$ to its reach is $\mathbf{P}_{i k}$.

For a node $i$ transmitting to node $j$, with nodes $\alpha_{0}, \alpha_{1}$, $\cdots, \alpha_{k}$ (arranged in order of increasing distance from $i$ ) covered implicitly, the decremental transmit power cost for letting node $j$ out of its reach is $\mathbf{P}_{i j}-\mathbf{P}_{i \alpha_{k}}$. If no node is covered implicitly, the decremental transmit power cost for letting node $j$ out of its reach is $\mathbf{P}_{i j}$.

- Criterion (2) ensures that the TTFF of refined tree is the same as the optimal TTFF obtained using the MDLT algorithm.

- Criterion (3) ensures that the transmitter power levels assigned to the nodes after the improvement phase do not violate the maximum power constraint.

- Criterion (4) ensures that the residual lifetimes of the participating nodes in the improved tree are greater than or equal to the session duration, $L / R$, where $L$ is the total number of bits to be transmitted and $R$ is the data rate in bits per second.

For example, assume that Figure 2(a) is the MDLT tree. Let node 6 be the critical node with an associated TTFF of $\hat{\tau}=E_{6} / \mathbf{P}_{64}$. If we apply the 1 -shrink operation to node 1 , resulting in the disconnected graph shown in Figure 3(a), the incremental cost of assigning node 5 as a child of node 4 is equal to $\mathbf{P}_{45}-\mathbf{P}_{41}$ and the decremental cost at node 1 for

\footnotetext{
${ }^{4}$ Note that restricting the foster parents to the set of the non-descendants, excluding its current parent, prevents the occurrence of cycles in the improved graph.
}

letting node 5 out of its reach is $\mathbf{P}_{15}$. Node 5 can be better accommodated from node 4 if all the following conditions are satisfied:
(a) $\mathbf{P}_{45}-\mathbf{P}_{41}<\mathbf{P}_{15}$
(b) $E_{4} / \mathbf{P}_{45} \geq \hat{\tau}=E_{6} / \mathbf{P}_{64}$
(c) $\mathbf{P}_{45} \leq P_{4}^{\max }$
(d) $E_{4} / \mathbf{P}_{45} \geq L / R$

where (b) is the TTFF violation check, (c) is the maximum power constraint and (d) is the session duration check. Note that only transmit power is accounted for in condition (a) since node 5 is a receiver in both the original tree (Figure 2a) and the improved tree (Figure $3 \mathrm{~b}$ ) and $P_{5}^{r x}$ is independent of the choice of the transmitting node.

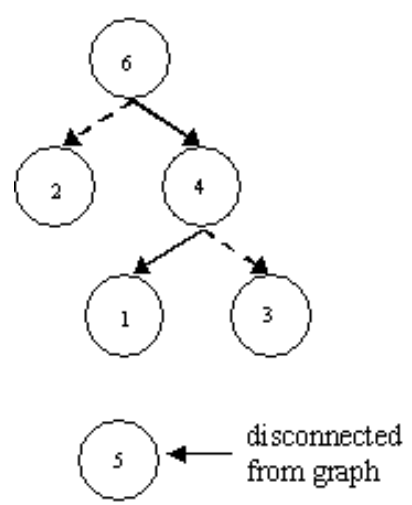

(a)

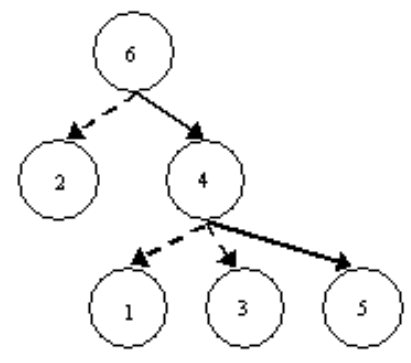

(b)
Fig. 3. (a) Temporarily disconnected graph as a result of applying the 1-shrink operation on node 1 in Figure 2. (b) Improved tree obtained by assigning node 5 as a child of node 1 .

In general, if $i \in c h(j)$ and $k \in f p a(i), i$ can be better accommodated from $k$ after applying a 1-shrink operation on $j$, if the following conditions are satisfied:

$$
\begin{aligned}
& \text { - } \Delta Y_{k}^{(+) t x}<\Delta Y_{j}^{(-) t x} \\
& \text { - } E_{k} / \mathbf{P}_{k i} \geq \hat{\tau} \text { and } E_{k} / \mathbf{P}_{k i} \geq L / R \\
& \text { - } \mathbf{P}_{k i} \leq P_{i}^{\max }
\end{aligned}
$$

where $\Delta Y_{k}^{(+) t x}$ is the incremental transmit power cost at node $k, \Delta Y_{j}^{(-) t x}$ is the decremental transmit power cost at node $j$ and $\hat{\tau}$ is the TTFF of the MDLT tree. If there is more than one foster parent better able to accommodate the temporarily disconnected child, the one which would lead to a maximum reduction in overall tree power is chosen to be the new parent. Ties, if any, are broken arbitrarily.

The sequence in which the candidate parent nodes are checked is bottom-up; i.e., parents at level (see Figure 2) $M A X_{-} L V L-1$ are checked first, followed by those at level 
$M A X_{-} L V L-2$, terminating with the source at level 0 . If an improvement is found at any step, the graph is modified by reassigning the temporarily disconnected child to its new foster parent and the algorithm is repeated on the new graph.

Figure 4 provides a high level description of the 1-shrink for MDLT algorithm.

1. Let $G$ be the $M D L T$ tree, represented as a digraph rooted at the source.

2. Set $M A X \_L V L=$ maximum level in $G$;

3. Set $l=M A X_{-} L V L-1$;

4. Set $\overrightarrow{p a}_{l}=$ set of candidate parent nodes at level $l$;

5. Set no_pa $=$ no. of candidate parent nodes at level $l=\left|\overrightarrow{p a}_{l}\right|$;

6. Set $n=1$;

7. Apply the 1-shrink operation to the candidate parent node $\overrightarrow{p a} a_{l}(n)$. Check whether its temporarily disconnected child, $i$, can be better accommodated from any node in the set $f p a(i)$ (see eqns. 16 and 17).

8. if(better accommodation possible)

- Identify the foster parent which will lead to a maximum reduction in tree cost. Modify $G$ by assigning this node to be the new parent of node $i$.

- Repeat steps 2 to 8 on the new $G$.

else

if $\left(n<n o_{-} p a\right)$

/* Not all candidate parent nodes checked at level $l * /$

- $n=n+1$;

- Repeat steps 7 and 8.

else

/* All candidate parent nodes checked at level $l * /$

if $(l>0)$

- $l=l-1$;

- Repeat steps 4 to 8 .

else

/* All candidate parent nodes checked at all levels */

Stop and print $G$. $/ *$ End of procedure */ endif

endif

endif

Fig. 4. High level description of the 1-shrink for MDLT algorithm.

\section{Vi. Simulation Results}

We conducted a study of the performance of optimal and heuristic methods for different multicast group sizes in 15, $30,50,75$ and 100 -node networks in a $10 \times 10$ grid. The channel loss exponent $\alpha$ (see eqn. 1 or 2) was set to 2 for all simulations. Receiver power consumption was equal to 0.01 , i.e., $\mathbf{P}_{j}^{r x}=0.01, \forall j \in \mathcal{N}$ in (2). The networks and the destination sets were chosen so that all destination nodes could be reached, given the transmitter power constraints. Transmitter power constraints were set so that each node was connected to its 4 nearest neighbors. The linear programming solver, LINDO [9], which uses a LP-based branch and brand algorithm to solve MILP problems, was used to compute the optimal solutions. In this section, we use the notation $H r_{1}$ to represent "without tree-improvement" heuristic solution (i.e., MDLT + pruning) and $\mathrm{Hr}_{2}$ to represent "with 1shrink tree-improvement" solution (i.e., MDLT + pruning + 1 -shrink for MDLT, in that order). Note that we do not compare the TTFF of the optimal and heuristic solutions since the tree-improvement algorithm does not affect the optimal TTFF.

Our performance measures for comparing the optimal and heuristic solutions are the mean $\left(P M_{1}\right), \max \left(P M_{2}\right)$ and standard deviation $\left(P M_{3}\right)$ of the quantity $100 \times \frac{A-B}{B}$. The significance of the parameters $A$ and $B$ is described below.

For $N=15$ and 30, we compare the performance of the 2step heuristic algorithm w.r.t the optimal, i.e., the parameters $A$ and $B$ are $\sum_{i} Y_{i}(H r 2)$ and $\sum_{i} Y_{i}(o p t)$ respectively. Table I provides a statistical summary of the simulation results for $N=15$ and 30 . It can be seen that, on average $\left(P M_{1}\right)$, the improved solution is within $1.5 \%$ of the optimal solution for all cases. The worst instance $\left(P M_{2}\right)$ we observed was for $N=30, D=9$, when the total transmitter power of the improved solution was approximately $30 \%$ higher than the optimal.

TABLE I

Comparison of optimal and heuristic solutions for $N=15$ and 30 .

\begin{tabular}{|c|c|c|c|c|}
\hline$N$ & $D$ & $P M_{1}$ & $P M_{2}$ & $P M_{3}$ \\
\hline \multirow{4}{*}{15} & 4 & 1.02 & 12.65 & 2.92 \\
& 8 & 0.23 & 9.55 & 1.36 \\
& 12 & 1.38 & 21.30 & 5.95 \\
\hline \multirow{3}{*}{30} & 3 & 0.13 & 5.01 & 0.71 \\
& 6 & 0.44 & 6.03 & 1.56 \\
& 9 & 1.44 & 28.86 & 5.45 \\
\hline
\end{tabular}

For $N=50,75$ and 100, we did not obtain the optimal solutions due to the long running times of the LP software. We therefore compare the performance of the heuristic algorithm with and without the tree improvement procedure. The parameters $A$ and $B$ for these network sizes are therefore $\sum_{i} Y_{i}(H r 2)$ and $\sum_{i} Y_{i}(H r 1)$ respectively. Table II summarizes the performance of the heuristic with and without the tree-improvement algorithm for all network sizes. It can be seen that the improvement obtained by refining the MDLT tree is close to $6 \%$ as the number of destination nodes increases for $N \geq 50$. Since the improvement algorithm searches for better accommodations only within a subset of the nodes existing in the initial tree, we conjecture that the reason why improvement is not so significant for smaller ra- 
TABLE II

Comparison of heuristic solutions with and without tree-improvement.

\begin{tabular}{|c|c|c|c|c|}
\hline$N$ & $D$ & $P M_{1}$ & $P M_{2}$ & $P M_{3}$ \\
\hline \multirow{4}{*}{15} & 4 & -1.58 & 69.17 & 11.47 \\
& 8 & -2.57 & 7.32 & 1.79 \\
& 12 & -4.32 & 15.29 & 4.39 \\
\hline \multirow{3}{*}{30} & 3 & -4.12 & 23.41 & 4.64 \\
& 6 & -2.32 & 8.89 & 1.95 \\
& 9 & -3.64 & 16.09 & 3.75 \\
\hline \multirow{4}{*}{50} & 5 & -3.97 & 16.70 & 3.94 \\
& 10 & -4.86 & 15.43 & 3.60 \\
& 15 & -5.31 & 16.78 & 3.52 \\
\hline \multirow{4}{*}{75} & 5 & -4.05 & 15.75 & 3.83 \\
& 10 & -5.41 & 20.96 & 4.12 \\
& 15 & -5.74 & 19.79 & 3.57 \\
\hline \multirow{3}{*}{100} & 5 & -5.62 & 28.06 & 5.43 \\
& 10 & -5.65 & 15.10 & 3.03 \\
& 15 & -6.72 & 14.60 & 3.46 \\
\hline
\end{tabular}

tios of $D / N$ is simply because the initial trees in such cases tend to be "short and thin", thereby limiting the set of feasible foster parents and the improvement search space.

\section{Conclusion}

We considered the problem of joint maximization of the time-to-first-failure (TTFF) and minimization of the total power in energy constrained static wireless networks. We proposed a 2-step heuristic procedure for the joint optimization problem. The first step is the MDLT algorithm which provides an optimal solution, but with respect to the TTFF criterion only. The second step is an improvement algorithm which is used to refine the MDLT tree such that the total transmit power is reduced, without affecting the optimal TTFF. Simulations conducted on various network sizes and multicast groups show that the improvement procedure leads to a significant reduction in overall tree power. For small networks, simulations show that the heuristic solutions are near-optimal.

\section{REFERENCES}

1) Jae-Hwan Chang and Leandros Tassiulas, "Energy Conserving Routing in Wireless Ad-Hoc Networks", Proc. of INFOCOM, 2000.

2) R. J. Marks II, A. K. Das, M. A. El-Sharkawi, P.Arabshahi and A. Gray, "Maximizing Lifetime in an Energy Constrained Wireless Sensor Array Using Team Optimization of Cooperating Systems", Proc. of the World Congress on Computational Intelligence, Honolulu, Hawaii, 2002.

3) Archan Misra and Suman Banerjee, "MRPC: Maximizing Network Lifetime for Reliable Routing in Wireless Environments", Proc. of WCNC, 2002.

4) Intae Kang and Radha Poovendran, "On the Lifetime Extension of Energy-Efficient Multihop Broadcast Networks", Proc. of the World Congress on Computational Intelligence, Honolulu, Hawaii, 2002.

5) C.K. Toh, "Maximum Battery Life Routing to support ubiquitous Mobile Computing in Wireless Ad Hoc Networks", IEEE Communications Magazine, June 2001.

6) A. K. Das, R.J. Marks II, M.A. El-Sharkawi, P. Arabshahi and A. Gray, "Maximization of Time-to-Firstfailure for Multicasting in Wireless Networks : Optimal Solution", Proc. of MILCOM, October 2004.

7) A. K. Das, R.J. Marks II, M.A. El-Sharkawi, P. Arabshahi and A. Gray, "MDLT: A Polynomial Time Optimal Algorithm for maximization of Time-to-FirstFailure in Energy-Constrained Broadcast Wireless Networks," Proc. of GLOBECOM, San Francisco, December 1-5, 2003.

8) A. K. Das, R.J. Marks II, M.A. El-Sharkawi, P. Arabshahi and A. Gray, " $r$-shrink: A Heuristic for Improving Minimum Power Broadcast Trees in Wireless Networks," Proc. of GLOBECOM, San Francisco, December 1-5, 2003.

9) LINDO LP/MILP solver, $<$ www.lindo.com $>$ 